\title{
The determination and enhancement of compliant modes for high-amplitude actuation in lattices
}

\author{
Zahra Soltani*, Matthew Santer \\ Department of Aeronautics, Imperial College London, London, SW7 2AZ, UK
}

\begin{abstract}
This paper details the nonlinear design of adaptive lattices by determination and enhancement of compliant modes and optimizing the designed structure for delivering high amplitude actuation. The particular focus is the kagome lattice geometry - a pattern with some unique and useful actuation properties. Developing a novel design tool, the stiffness matrix of the beam assembly is calculated using a developed second-order geometrically nonlinear beam finite element formulation allowing large rotations. Based on this formulation in conjunction with singular value decomposition of the stiffness matrix, the modal optimization technique reduces the continuous structure with many degrees of freedom to a small number of low energy modes, which form the basis of designing the adaptive structure. For delivering high-amplitude actuation, the designed structure needs to be re-optimized due to changes in the nonlinear stiffness matrix under large deformation. This is performed via Bayesian optimization and by removing some internal members of the lattice. The integrity and feasibility of the optimum design is guaranteed via defining some constraints on removed members.
\end{abstract}

Keywords: Adaptive structures, Large deformation, Nonlinear finite element, Bayesian optimization, Kagome lattice

2010 MSC: 00-01, 99-00

\footnotetext{
* Corresponding author

Email address: Z.Soltani@imperial.ac.uk (Zahra Soltani)
}

Preprint submitted to International Journal of Solids and Structures 


\section{Introduction}

Lattice structures can be analysed in two main categories, depending on whether the lattices are formed with members having pin-joined or rigid-joined connections. The adaptive behaviour of a pin-joined framework is fully understood

5 in terms of its kinematic and static indeterminacies, which are defined as the modes of inextensional deformation and the states of self-stress, respectively. In turn, infinitesimal inextensional mechanisms tighten up with the higher orders of displacement which have a detrimental effect on the adaptivity of the assembly [1]. McCallen and Romstad [2] developed an equivalent continuum model for the geometrically nonlinear analysis of beam-like lattice structures, by requiring the strain energy of the continuum equal to the strain energy of the lattice for a finite set of assumed deformation modes. Cao et al. [3] extended the continuum model to nonlinear beam-like lattice truss structures by introducing a co-rotational coordinate system to decompose the motion into rigid body motion and pure deformations. Generalized quasicontinuum modeling of metallic lattices with geometrical and material nonlinearity and variability is studied in Ref [4]. Jamshidian et al. [5] investigated the large-deformation nonlinear elastic behaviour of beam-lattice metamaterials by micromechanical nonlinear buckling analysis, where the effective constitutive behaviour of representative unit cells is derived by the uniaxial standard test. In this paper we consider the design and optimization of lattice structure subject to high amplitude actuation. Based on an FE discretization method and using the modal reduction technique, the design of compliant lattice is performed via determination and enhancement of its compliant modes. Under high amplitude actuation, the effectiveness of 25 the designed lattice reduces significantly due to the induced internal stresses. This implies the necessity of re-optimizing the designed lattice based on the functionality of the structure for delivering the desired actuation response.

The developed formulation is general, but the present work focuses on the so-called kagome lattice, with a representative hexagonal unit cell shown in Fig. so 1(a). The kagome lattice has high potential for use in lightweight structures 
due to its high relative stiffness and strengths. Specifically, compared to the triangular lattice, in which the members are intersecting at the center of the hexagonal unit cell (Fig. 1(b)), the kagome lattice with the same relative density has four-times greater bucking strength [6].

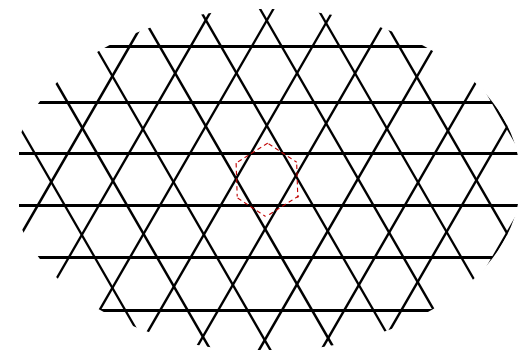

(a)

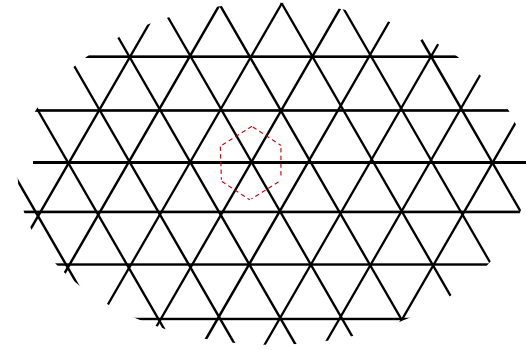

(b)

Figure 1: Infinite repetitive (a) kagome and (b) triangular lattice structures with a representative hexagonal unit cell

We denote each row as an actuation 'corridor'. Kagome lattices possess some unique and attractive actuation properties in the laterally-confined and farreaching deformation along each corridor via the actuation of a single member [7]. These characteristic make kagome lattices suitable for the production of in-plane surface waves of variable wave length. The principle is demonstrated

in Fig. 2 where adjacent corridors are driven with different amplitudes to create two waves of different lengths. A quasi-stiffness matrix is presented by Bird et al.[8] for determination of the compliant modes of a kagome lattice and the subsequent optimization of the lattice by manipulating these modes.

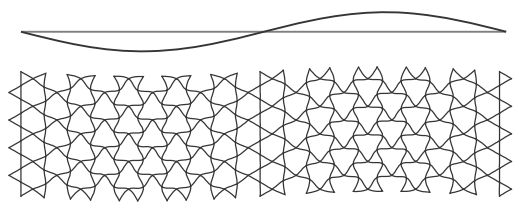

(a) Large wavelength

Figure 2: Using kagome lattices for producing in-plane waves of variable wave length [9]

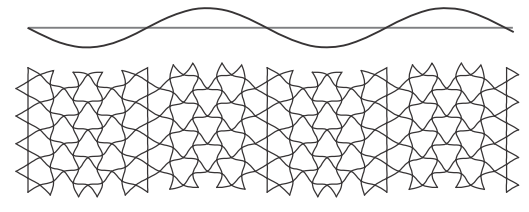

(b) Short wavelength

In an experimental study[9], a novel compliant adaptive surface was cre45 ated and measured, via attaching the kagome lattice to a membrane skin, to be capable of generating controllable in-plane travelling waves in a structurally 
supported continuum. The innovative surface has been shown experimentally to be capable of producing waveforms of variable length and speed and recording a significant reduction in skin friction[10]. However in previous works designs were predominately based on a linear formulation which is valid only for the design of lattices under small amplitude actuation. The present work which employs a novel nonlinear formulation in conjunction with Bayesian optimization, presents a bespoke design tool for the optimum design of lattices with a significant reduction in the actuation force. A large deformation theory for in-plane beam problems, analogous to Budiansky's nonlinear shell theory, is formulated by Epstine and Murrey [11]. The geometrically nonlinear finite element formulation of elastic beams in this work is derived for the general 3D load condition. The formulation stems from a second-order geometric approximation for finite rotations presented by Argyris [12] and was used for developing a nonlinear layer-wise shell element formulation in the study of compliant composite morphing wings [13], [14]. The developed formulation is applied in an in-house code and compliance modes of the lattice are extracted using the singular value decomposition as a model reduction technique due to the cost benefits of computing and complexity under high-amplitude actuation. The routine is used as 65 a design tool to refine the kagome lattice by determination and enhancement of some key compliant modes for delivering desired actuation modes. However, it is shown that compliance modes change significantly under high-amplitude actuation and subsequently reduce the functionality of the designed structure. This implies the necessity of the re-optimization of the adaptive structure under large deformation. This is performed using a machine-learning-based optimization method. The process illustrates how by removing some internal members subject to feasibility constraints, a modified integrated feasible design of the lattice can be obtained which is effective for high-amplitude actuation. 


\section{Nonlinear second order beam finite element formulation}

75 In this section, a second-order kinematic and geometrically nonlinear finite element formulation of elastic beams is derived under large displacements and rotations, with the assumption of small strain values.

\subsection{Second-order kinematics of elastic beams under large rotations}

Figure 3 shows the motion of a beam in a stationary Cartesian coordinate system, where it can experience large displacements and rotations with small strains. The convected orthogonal coordinate system at each point, ${ }^{t} x_{i}(i=$ $1,2,3)$, is defined along the principle axes of beam at time $t$, where ${ }^{t} \hat{\boldsymbol{e}}_{3}$ denotes the unit tangential vector along the beam axis. In the following, a right subscript $i=1,2,3$ indicates that the variables apply to the convected, rather than the global coordinate system. Also, the left superscript is used to denote the time that the variable value is measured.

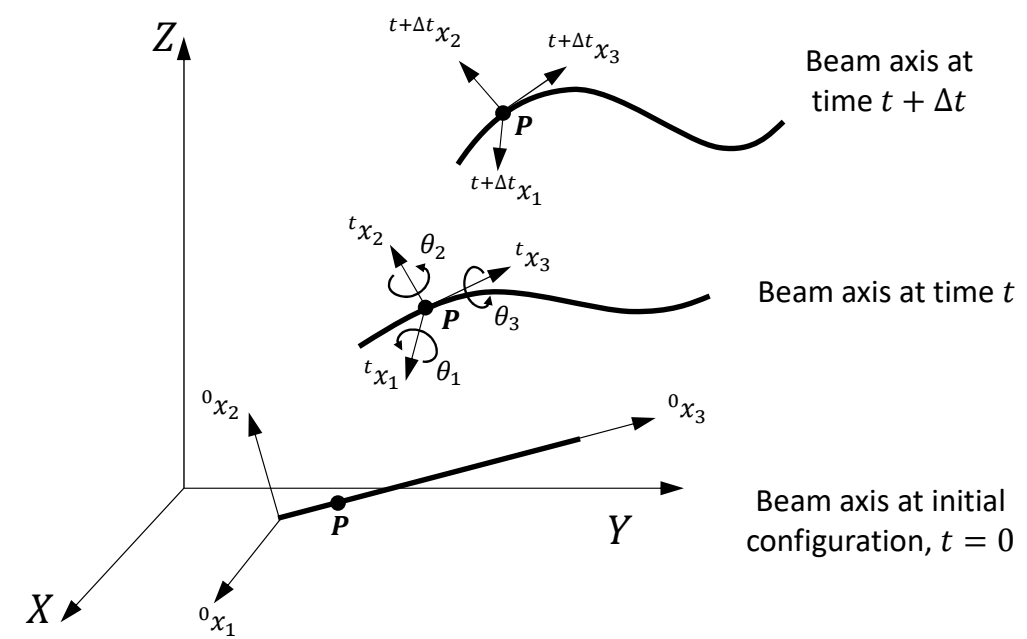

Figure 3: Motion of beam axis in Cartesian coordinate frame

The main assumptions in this study are as follows:

1. The kinematic conditions do not include warping deformation (i.e., plane sections remain plane); 
2. The strains normal to the beam axis are negligible (i.e., the cross section areas remain constant).

The kinematics of the beam are studied based on the Updated Lagrangian (UL) incremental approach, which has more numerical effectiveness in comparison with the Total Lagrangian (TL) formulation[15]. In the UL framework, referring to Fig. 3, the position vector of a generic point $\boldsymbol{P}$ along the beam axis at a known configuration of the beam at time $t$ can be described as

$$
{ }^{t} \boldsymbol{r}=x_{3}{ }^{t} \hat{\boldsymbol{e}}_{3}
$$

Then, the position vector of a point in the corresponding cross section is expressed as

$$
{ }^{t} \boldsymbol{R}={ }^{t} \boldsymbol{r}+x_{1}{ }^{t} \hat{\boldsymbol{e}}_{1}+x_{2}{ }^{t} \hat{\boldsymbol{e}}_{2}
$$

During motion of the beam to an unknown configuration at time $t+\Delta t$, making use of assumptions 1 and 2, this same point deforms to

$$
{ }^{t+\Delta t} \boldsymbol{R}={ }^{t+\Delta t} \boldsymbol{r}+x_{1}{ }^{t+\Delta t} \hat{\boldsymbol{e}}_{1}+x_{2}{ }^{t+\Delta t} \hat{\boldsymbol{e}}_{2}
$$

where ${ }^{t+\Delta t} \boldsymbol{r}={ }^{t} \boldsymbol{r}+\boldsymbol{W}$, in which $\boldsymbol{W}\left(x_{3}\right)=\left\{\begin{array}{lll}w_{1} & w_{2} & w_{3}\end{array}\right\}^{T}$ expresses the incremental displacement vector of the beam axis and is measured in the convected coordinate system at time $t$. If the incremental finite rotation components around the principle axes of beam ${ }^{t} \hat{\boldsymbol{e}}_{i}$ at the generic point $\boldsymbol{P}$ in Fig. 3 are 105 denoted with $\boldsymbol{\Theta}\left(x_{3}\right)=\left\{\begin{array}{lll}\theta_{1} & \theta_{2} & \theta_{3}\end{array}\right\}^{T}$, the updated director vectors after some geometric manipulations can be expressed as

$$
{ }^{t+\Delta t} \hat{\boldsymbol{e}}_{i}=\boldsymbol{\Theta} \times{ }^{t} \hat{\boldsymbol{e}}_{i}+\frac{1}{2} \boldsymbol{\Theta} \times\left(\boldsymbol{\Theta} \times{ }^{t} \hat{\boldsymbol{e}}_{i}\right)
$$

Further details concerning this relation can be found in Ref [12]. Using Eq (4), the new transverse director vectors are obtained as 


$$
\begin{aligned}
{ }^{t+\Delta t} \hat{\boldsymbol{e}}_{1} & =\left\{\begin{array}{lll}
1-\frac{1}{2}\left(\theta_{2}^{2}+\theta_{3}^{2}\right) & \theta_{3}+\frac{1}{2} \theta_{1} \theta_{2} & -\theta_{2}+\frac{1}{2} \theta_{1} \theta_{3}
\end{array}\right\}^{T} \\
{ }^{t+\Delta t} \hat{\boldsymbol{e}}_{2} & =\left\{\begin{array}{lll}
-\theta_{3}+\frac{1}{2} \theta_{1} \theta_{2} & 1-\frac{1}{2}\left(\theta_{1}^{2}+\theta_{3}^{3}\right) & \theta_{1}+\frac{1}{2} \theta_{2} \theta_{3}
\end{array}\right\}^{T}
\end{aligned}
$$

Substituting Eqs (4a)-(4b) in Eq (3) and subtracting Eq (1) from the result, yields the following second-order approximations for increments in the displacement field with finite rotations from time $t$ to time $t+\Delta t$.

$$
\begin{array}{r}
u_{1}\left(x_{1}, x_{2}, x_{3}\right)=w_{1}\left(x_{3}\right)-x_{2} \theta_{3}\left(x_{3}\right)+\frac{x_{2}}{2} \theta_{1} \theta_{2}-\frac{x_{1}}{2}\left(\theta_{2}^{2}+\theta_{3}^{2}\right) \\
u_{2}\left(x_{1}, x_{2}, x_{3}\right)=w_{2}\left(x_{3}\right)+x_{1} \theta_{3}\left(x_{3}\right)+\frac{x_{1}}{2} \theta_{1} \theta_{2}-\frac{x_{2}}{2}\left(\theta_{1}^{2}+\theta_{3}^{2}\right) \\
u_{3}\left(x_{1}, x_{2}, x_{3}\right)=w_{3}\left(x_{3}\right)-x_{1} \theta_{2}\left(x_{3}\right)+x_{2} \theta_{1}\left(x_{3}\right)+\frac{x_{1}}{2} \theta_{1} \theta_{3}+\frac{x_{2}}{2} \theta_{2} \theta_{3}
\end{array}
$$

It is worth noting that the first linear terms in $\operatorname{Eqs}(5)$ are in accordance with the well-known first order displacement field for an Euler-Bernoulli beam [16].

Large deformation with small strain values during bending deformation of the beam structure, calls for the Green-Lagrange (GL) strain tensor that is invariant under rigid body rotations. The incremental components of GL strain in the convected coordinate system are given by

$$
\epsilon_{i j}=\frac{1}{2}\left(u_{i, j}+u_{j, i}+u_{k, i} u_{k, j}\right)
$$

where a comma denotes differentiation with respect to the following coordinates. Introducing Eqs (5) into the above equation and ignoring the third and higherorder terms, the non-zero components of incremental GL strains are obtained by 


$$
\begin{aligned}
\epsilon_{33} & =w_{3}^{\prime}-x_{1} \theta_{2}^{\prime}+x_{2} \theta_{1}^{\prime}+\frac{\theta_{3}}{2}\left(x_{1} \theta_{1}^{\prime}+x_{2} \theta_{2}^{\prime}\right)+\frac{\theta_{3}^{\prime}}{2}\left(x_{1} \theta_{1}+x_{2} \theta_{2}\right) \\
& +\frac{1}{2}\left[\left(w_{1}^{\prime}-x_{2} \theta_{3}^{\prime}\right)^{2}+\left(w_{2}^{\prime}+x_{1} \theta_{3}^{\prime}\right)^{2}+\left(w_{3}^{\prime}-x_{1} \theta_{2}^{\prime}+x_{2} \theta_{1}^{\prime}\right)^{2}\right] \\
2 \epsilon_{13} & =w_{1}^{\prime}-x_{2} \theta_{3}^{\prime}-\theta_{2}-x_{1}\left(\theta_{2} \theta_{2}^{\prime}+\theta_{3} \theta_{3}^{\prime}\right)+\frac{x_{2}}{2}\left(\theta_{1} \theta_{2}^{\prime}+\theta_{1}^{\prime} \theta_{2}\right)+\frac{1}{2} \theta_{1} \theta_{3} \\
& +\theta_{3}\left(w_{2}^{\prime}+x_{1} \theta_{3}^{\prime}\right)-\theta_{2}^{\prime}\left(w_{3}^{\prime}-x_{1} \theta_{2}^{\prime}+x_{2} \theta_{1}^{\prime}\right) \\
2 \epsilon_{23} & =w_{2}^{\prime}+x_{1} \theta_{3}^{\prime}+\theta_{1}+\frac{x_{1}}{2}\left(\theta_{1} \theta_{2}^{\prime}+\theta_{1}^{\prime} \theta_{2}\right)-x_{2}\left(\theta_{1} \theta_{1}^{\prime}+\theta_{3} \theta_{3}^{\prime}\right)+\frac{1}{2} \theta_{2} \theta_{3} \\
& -\theta_{3}\left(w_{1}^{\prime}-x_{2} \theta_{3}^{\prime}\right)+\theta_{1}^{\prime}\left(w_{3}^{\prime}-x_{1} \theta_{2}^{\prime}+x_{2} \theta_{1}^{\prime}\right)
\end{aligned}
$$

where the prime symbol is used to denote $x_{3}$-derivatives.

\subsection{Geometrically nonlinear FE formulation}

Figure 4 shows a beam element with two nodes at two consecutive time steps which undergoes a general three dimensional displacement field.
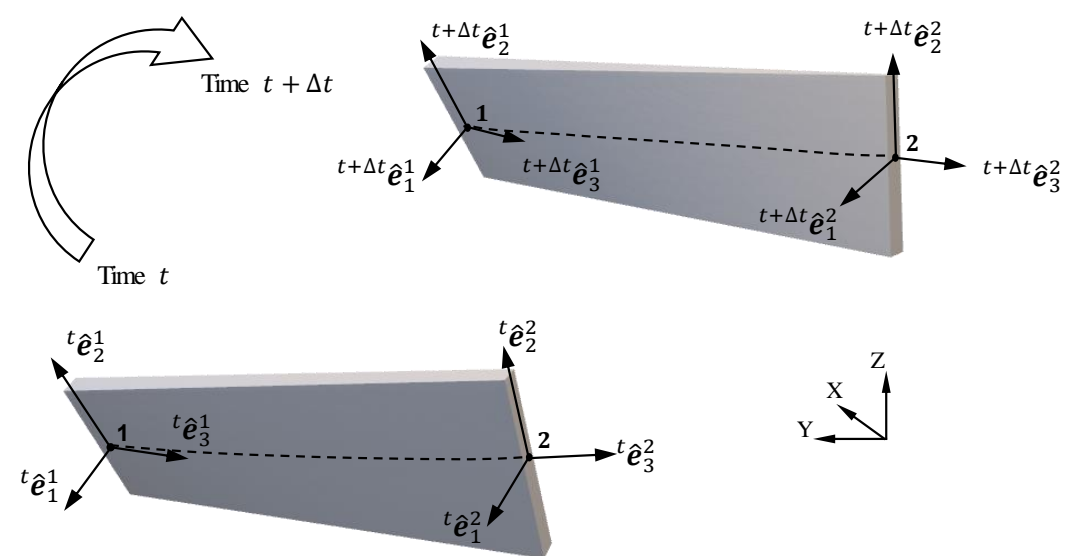

Figure 4: Beam element under large deformation

Each node has six degrees of freedom, consisting of three displacements and three rotations. The incremental displacement vector at node $k$ in the ${ }_{125}$ convected local coordinate system is expressed as $\boldsymbol{U}^{k}=\left\{\begin{array}{lllll}w_{1}^{k} w_{2}^{k} & w_{3}^{k} & \theta_{1}^{k} & \theta_{2}^{k} & \theta_{3}^{k}\end{array}\right\}^{T}$. Transformation between the local and global Cartesian coordinate systems can be performed using the updated director vectors at time $t$ as follows. 


$$
\boldsymbol{q}^{k}=\left[\begin{array}{cc}
{ }^{t} \boldsymbol{T}^{K} & \mathbf{0} \\
\mathbf{0} & \boldsymbol{T}^{k}
\end{array}\right] \boldsymbol{U}^{k}
$$

where

$$
{ }^{t} \boldsymbol{T}^{k}=\left[\begin{array}{lll}
{ }^{t} \hat{\boldsymbol{e}}_{1} & { }^{t} \hat{\boldsymbol{e}}_{2} & { }^{t} \hat{\boldsymbol{e}}_{2}
\end{array}\right]
$$

and $\boldsymbol{q}^{k}$ is the incremental displacement vector at node $k$ in the global Cartesian coordinate system.

The finite element formulation is implemented using linear interpolations to describe axial displacement and torsional rotation, and Hermitian functions to interpolate the transverse bending displacements and rotations. Then, with respect to the right-handed convected coordinate system defined in Fig. 4, the interpolations are as follows:

$$
\begin{aligned}
& w_{1}=h_{3} w_{1}^{1}+h_{4} \theta_{2}^{1}+h_{5} w_{1}^{2}+h_{6} \theta_{2}^{2} \\
& w_{2}=h_{3} w_{2}^{1}-h_{4} \theta_{1}^{1}+h_{5} w_{2}^{2}-h_{6} \theta_{1}^{2} \\
& w_{3}=h_{1} w_{3}^{1}+h_{2} w_{3}^{2} \\
& \theta_{1}=-h_{3}^{\prime} w_{2}^{1}+h_{4}^{\prime} \theta_{1}^{1}-h_{5}^{\prime} w_{2}^{2}+h_{6}^{\prime} \theta_{1}^{2} \\
& \theta_{2}=h_{3}^{\prime} w_{1}^{1}+h_{4}^{\prime} \theta_{2}^{1}+h_{5}^{\prime} w_{1}^{2}+h_{6}^{\prime} \theta_{2}^{2} \\
& \theta_{3}=h_{1} \theta_{3}^{1}+h_{2} \theta_{3}^{2}
\end{aligned}
$$

where the relations for linear and Hermitian shape functions $h_{i}$ are given in the Appendix.

In the development of the geometrically nonlinear finite element formulation in the UL framework, the equilibrium of the body at time $t+\Delta t$ is expressed by employing the principle of virtual displacements as follows

$$
\int_{{ }^{t} V}{ }^{t+\Delta t}{ }^{t} S_{i j} \delta_{t}^{t+\Delta t} \epsilon_{i j} d^{t} V={ }^{t+\Delta t} \mathfrak{R}
$$

135 where ${ }^{t+\Delta t} \mathfrak{R}$ is the external virtual work. Also, ${ }_{t}^{t+\Delta t} S$ denotes the 2nd-Piola Kirchhoff (PK2) stress tensor, which is the work-conjugate with the GL strain 
tensor [17] and is related to the Cauchy stress tensor by

$$
{ }^{t+\Delta t} S_{i j}=\frac{{ }^{t} \rho}{t+\Delta t} \rho{ }^{t+\Delta t} x_{i, m}{ }^{t+\Delta t} \sigma_{m n}{ }_{t}^{t+\Delta t} x_{j, n}
$$

where ${ }_{t}^{t+\Delta t} x_{i, j}=\partial^{t+\Delta t} x_{i} / \partial^{t} x_{j}$ is the deformation gradient tensor, which describes the stretches and rotations that material fibers undergo from time $t$ to time $t+\Delta t$.

By analogy to the notation used for coordinates in Section 2.1, the left superscript indicates in which configuration the quantity occurs. In addition, the left subscript indicates the configuration with respect to which the quantity is measured. Also, a quantity without a left superscript and subscript, refers to the incremental value, similar to the notation used for displacement and strain fields in Eqs (5) and (7), respectively.

Satisfying the equilibrium equation (11) through the complete history of load application, the large deformation of compliant structures is determined via a step-by step solution and using incremental decompositions of PK2 stress and GL strain tensors in UL framework, i.e. ${ }_{t}^{t+\Delta t} S_{i j}={ }^{t} \sigma_{i j}+{ }_{t} S_{i j}$ and ${ }_{t}^{t+\Delta t} \epsilon_{i j}={ }_{t} \epsilon_{i j}$. In each increment, since the internal force and stiffness matrix depend on the nodal displacements, an iterative solution scheme should be applied. In this fashion, the incremental finite element form of equilibrium Eq (11) using the Newton-Raphson method can be expressed as follows

$$
\begin{aligned}
\left({ }_{t}^{t+\Delta t} \boldsymbol{K}_{L}^{(i-1)}+{ }_{t}^{t+\Delta t} \boldsymbol{K}_{N L}^{(i-1)}\right) \boldsymbol{q}^{(i)} & ={ }^{t+\Delta t} \boldsymbol{R}-{ }_{t}^{t+\Delta t} \boldsymbol{F}^{(i-1)} \\
{ }^{t+\Delta t} \boldsymbol{q}^{(i)} & ={ }^{t+\Delta t} \boldsymbol{q}^{(i-1)}+\boldsymbol{q}^{(i)}
\end{aligned}
$$

where

$$
\begin{aligned}
& { }_{t}^{t+\Delta t} \boldsymbol{K}_{L}^{(i-1)}={ }^{t+\Delta t} \boldsymbol{T}^{(i-1)}{ }_{t}^{t+\Delta t} \boldsymbol{K}_{L}^{e t+\Delta t} \boldsymbol{T}^{(i-1)^{T}} \\
& { }^{t+\Delta t} \boldsymbol{K}_{N L}^{(i-1)}={ }^{t+\Delta t} \boldsymbol{T}^{(i-1)}{ }_{t}^{t+\Delta t} \boldsymbol{K}_{N L}^{e^{(i-1)} t+\Delta t} \boldsymbol{T}^{T^{(i-1)}} \\
& { }^{t+\Delta t} \boldsymbol{F}^{(i-1)}={ }^{t+\Delta t} \boldsymbol{T}^{(i-1)}{ }_{t}^{t+\Delta t} \boldsymbol{F}^{e^{(i-1)}}
\end{aligned}
$$


in which ${ }^{t+\Delta t} \boldsymbol{T}^{(i-1)}$ is the transformation matrix from the local element coordinate systems to global one which is formed from individual sub-matrices $\mathrm{Eq}(9)$, in which the director vectors at each iteration are updated using Eqs(4). Also, the element linear and nonlinear stiffness matrices and internal force vector in local coordinate system are obtained as follows

$$
\begin{aligned}
{ }_{t}^{t+\Delta t} \boldsymbol{K}_{L}^{e} & =\int_{V}{ }_{t}^{t+\Delta t} \boldsymbol{B}_{L}^{e^{T}}{ }_{t} \boldsymbol{C}^{e}{ }_{t}^{t+\Delta t} \boldsymbol{B}_{L}^{e} d V \\
{ }_{t}^{t+\Delta t} \boldsymbol{K}_{N L}^{e} & =\int_{V}{ }_{t}^{t+\Delta t} \boldsymbol{B}_{N L}^{e^{T}}{ }^{t} \overline{\boldsymbol{\sigma}}^{e}{ }_{t}^{t+\Delta t} \boldsymbol{B}_{N L}^{e} d V \\
{ }_{t}^{t+\Delta t} \boldsymbol{F}^{e} & =\int_{V}{ }^{t+\Delta t}{ }^{t+\Delta}{ }_{L}^{e^{T}}{ }^{t} \hat{\boldsymbol{\sigma}}^{e} d V
\end{aligned}
$$

where $\boldsymbol{B}_{L}^{e}$ and $\boldsymbol{B}_{N L}^{e}$ are linear and nonlinear strain-displacement matrices in element coordinate system, which are calculated by substituting the secondorder incremental displacement field Eqs (5) and the interpolations Eqs (10) in linear, $e_{i j}=\frac{1}{2}\left(u_{i, j}+u_{j, i}\right)$, and nonlinear, $\eta_{i j}=\frac{1}{2} u_{k, i} u_{k, j}$, parts of GL strain 165 Eq (6). Denoting ${ }_{t}^{t+\Delta t} \boldsymbol{B}^{e}=\left[{ }_{t}^{t+\Delta t} \boldsymbol{B}^{e^{1}} \mid{ }_{t}^{t+\Delta t} \boldsymbol{B}^{e^{2}}\right]$ for both linear and nonlinear matrices, they can be expressed after some mathematical manipulation as 
${ }_{t}^{t+\Delta t} \boldsymbol{B}_{L}^{e^{i}}=\left[\begin{array}{ccc}-x_{1} h_{(2 i+1)}^{\prime \prime}+x_{2} g_{(2 i+1)}^{\prime} & -x_{2} h_{(2 i+1)}^{\prime \prime}+x_{1} g_{(2 i+1)}^{\prime} & 0 \ldots \\ g_{(2 i+1)}+{ }_{[-2,2]}^{[1,1]} \Gamma_{(2 i+1):[1,2]}^{[2,4]} & \begin{array}{c}{[2,2,1]} \\ {[-2,1,-2]}\end{array} \Gamma_{(2 i+1):[1,2,2]}^{[2,4,5]} & 0 \ldots \\ { }_{[1,2,2,1]}^{[1,-2,2,-1]} \Gamma_{(2 i+1):[1,1,2,2]}^{[1,2,4,5]} & -g_{(2 i+1)}+{ }_{[-2]}^{[2]} \Gamma_{(2 i+1):[2]}^{[5]} & 0 \ldots \\ \ldots x_{2} h_{(2 i+2)}^{\prime \prime}+x_{1} g_{(2 i+2)}^{\prime} & -x_{1} h_{(2 i+2)}^{\prime \prime}+x_{2} g_{(2 i+2)}^{\prime} & 0 \\ \ldots{ }_{[-2,2]}^{[2,1]} \Gamma_{(2 i+2):[2,2]}^{[4,5]} & g_{(2 i+2)} & x_{1} h_{i}^{\prime}+{ }_{[-2]}^{[2]} \Gamma_{i:[-k]}^{[6]} \\ \ldots g_{(2 i+2)}+{ }_{[2]}^{[2]} \Gamma_{(2 i+2):[2]}^{[5]} & { }_{[-2]}^{[1]} \Gamma_{(2 i+2):[2]}^{[5]} & -x_{2} h_{i}^{\prime}+{ }_{[1-2]}^{[1]} \Gamma_{i:[-k]}^{[6]}\end{array}\right]$

$$
{ }_{t}^{t+\Delta t} \boldsymbol{B}_{N L}^{e^{i}}=\left[\begin{array}{cccccc}
0 & 0 & 0 & 0 & 0 & 0 \\
0 & 0 & 0 & 0 & 0 & -h_{i} \\
h_{2 i+1}^{\prime} & 0 & 0 & 0 & h_{2 i}^{\prime} & -x_{2} h_{i}^{\prime} \\
0 & 0 & 0 & 0 & 0 & h_{i} \\
0 & 0 & 0 & 0 & 0 & 0 \\
0 & h_{2 i+1}^{\prime} & 0 & -h_{2 i}^{\prime} & 0 & x_{1} h_{i}^{\prime} \\
-h_{2 i+1}^{\prime} & 0 & 0 & 0 & -h_{2 i}^{\prime} & 0 \\
0 & -h_{2 i+1}^{\prime} & 0 & h_{2 i}^{\prime} & 0 & 0 \\
-x_{1} h_{2 i+1}^{\prime \prime} & -x_{2} h_{2 i+1}^{\prime \prime} & h_{i}^{\prime} & x_{2} h_{2 i}^{\prime \prime} & -x_{1} h_{2 i}^{\prime \prime} & 0
\end{array}\right]
$$

where

$$
{ }_{\left[b_{1} \ldots b_{n}\right]}^{\left[a_{1} \ldots a_{n}\right]} \Gamma_{m:\left[d_{1} \ldots d_{n}\right]}^{\left[c_{1} \ldots c_{n}\right]}=\sum_{j=1}^{n} \sum_{k=1}^{\# \text { Node }} \frac{x_{a_{j}}}{b_{j}} \boldsymbol{U}_{\left(6(k-1)+c_{j}\right)}^{e}\left(h_{m}^{\prime} h_{2 k+d_{j}}^{\prime \prime}+h_{2 k+d_{j}}^{\prime} h_{m}^{\prime \prime}\right)
$$


and

$$
g_{m}=\frac{1}{2} h_{m}^{\prime}\left(h_{1} \theta_{3}^{1}+h_{2} \theta_{3}^{2}\right)
$$

In addition, terms ${ }_{t} \boldsymbol{C}^{e}$ and ${ }^{t} \hat{\boldsymbol{\sigma}}$ in Eqs (15a) and (15c) are the incremental stress-strain tensor and the Cauchy stress vector in the element coordinate system at time $t$, respectively. Also, ${ }^{t} \overline{\boldsymbol{\sigma}}^{e}$ in Eq (15b) denotes the Cauchy stress matrix in the following form.

$$
{ }^{t} \overline{\boldsymbol{\sigma}}^{e}=\left[\begin{array}{ccc}
{ }^{t} \boldsymbol{\sigma}^{e} & \mathbf{0} & \mathbf{0} \\
\mathbf{0} & { }^{*} \boldsymbol{\sigma}^{*} & \mathbf{0} \\
\mathbf{0} & \mathbf{0} & { }^{t} \boldsymbol{\sigma}^{*}
\end{array}\right]
$$

where

$$
{ }^{t} \boldsymbol{\sigma}^{*}=\left[\begin{array}{ccc}
{ }^{t} \sigma_{11}^{e} & { }^{t} \sigma_{12}^{e} & { }^{t} \sigma_{13}^{e} \\
{ }^{t} \sigma_{21}^{e} & { }^{t} \sigma_{22}^{e} & { }^{t} \sigma_{23}^{e} \\
{ }^{t} \sigma_{31}^{e} & { }^{t} \sigma_{32}^{e} & { }^{t} \sigma_{33}^{e}
\end{array}\right]
$$

For reasons of simplicity, the superscript $(i-1)$ is dropped from $\operatorname{Eq}(15)$ in subsequent formulation.

\section{Determination and enhancement of compliant modes}

175 The singular value decomposition (SVD) decomposes the stiffness matrix with $n$ degrees of freedom into its singular values according to

$$
\begin{aligned}
\overbrace{\boldsymbol{K}}^{(n \times n)} & =\overbrace{\boldsymbol{K}_{L}}^{(n \times n)}+\overbrace{\boldsymbol{K}_{N L}}^{(n \times n)}=\overbrace{\boldsymbol{\Psi}}^{(n \times n)} \overbrace{\boldsymbol{\Omega}}^{(n \times n)} \overbrace{\boldsymbol{\Phi}^{T}}^{(n \times n)} \\
& =\left[\begin{array}{cccc}
\mid & \mid & & \mid \\
\psi_{1} & \psi_{2} & \ldots & \psi_{n} \\
\mid & \mid & & \mid
\end{array}\right]_{(n \times n)}\left[\begin{array}{cccc}
\omega_{1} & & 0 & \\
& \omega_{2} & & \\
& & \ddots & \\
& 0 & & \omega_{n}
\end{array}\right]_{(n \times n)}\left[\begin{array}{lll}
- & \phi_{1}^{T} & - \\
- & \phi_{2}^{T} & - \\
\vdots & \\
- & \phi_{n}^{T} & -
\end{array}\right]_{(n \times n)}
\end{aligned}
$$

where the matrix $\boldsymbol{\Omega}$ is the diagonal matrix of singular values sorted in decreas- 
ing order, $\omega_{1} \geq \omega_{2} \geq \ldots \geq \omega_{n} \geq 0$. The matrices $\boldsymbol{\Psi}$ and $\boldsymbol{\Phi}$ are orthogonal matrices containing the left and right singular vectors. Then, from Eq (13), the incremental displacement of structure can be obtained by summation of inverted singular values and their compliant modes at time $t$, as

$$
\boldsymbol{q}=\boldsymbol{\Psi} \boldsymbol{\Omega}^{-1} \boldsymbol{\Phi}^{T}\left({ }^{t+\Delta t} \boldsymbol{R}-{ }^{t} \boldsymbol{F}\right)=\sum_{i=1}^{n} \frac{1}{\omega_{i}}\left\{\boldsymbol{\psi}_{i} \boldsymbol{\psi}_{i}^{T}\left({ }^{t+\Delta t} \boldsymbol{R}-{ }^{t} \boldsymbol{F}\right)\right\}
$$

where for the square, symmetric and positive definite stiffness matrices $\boldsymbol{\Psi}=\boldsymbol{\Phi}$, contains the compliant modes $\boldsymbol{\psi}_{i}$. As it can be observed from Eq (23), the SVD provides a model reduction technique in which the smallest singular values of $\boldsymbol{K}$ and their compliant modes have the most contribution to the incremental deformation.

In the following section, the generally applicable formulation detailed above is applied for the determination and enhancement of compliant modes of kagome lattice geometry.

\section{Kagome lattice design}

The work presented in this paper details the design of the compliant lattice for delivering large amplitude actuation based on the kagome geometry. Figure 5 shows a finite kagome lattice with six corridors $\left(r_{1}-r_{6}\right)$ and nineteen columns $\left(c_{1}-c_{19}\right)$. The internal geometry of the lattice is fully parameterized by the values of height, $d_{1}$, thickness $d_{2}$ and length, $d_{3}$, of the member. 


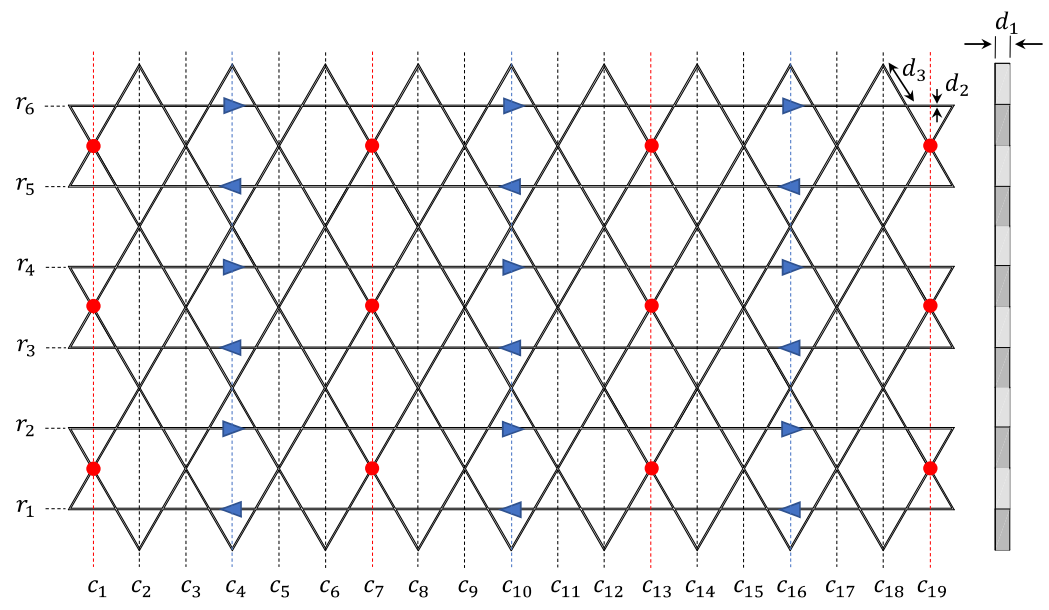

Figure 5: Finite kagome lattice structure defined with geometrical parameters: width $d_{1}$, thickness $d_{2}$ and length $d_{3}$

The kagome lattice is loaded at three actuation points along each corridor, shown using blue triangular symbols in Fig. 5, where the in-plane load direction changes alternately between two adjacent corridors. Also, boundary conditions are applied between actuating corridors, shown with filled-in red circles, to suppress out-of-plane compliant modes. To get large values of deformation, standing leaf springs are used between corridors as supporting flexible boundary conditions. More details on the compliant boundary conditions are discussed in Section 4.2. Also, the kagome lattice is given material properties corresponding to fully hardened AISI 301 graded stainless steel with Young's modulus $E=186$ GPa and Poisson ratio $\nu=0.3$.

\subsection{Geometric design}

The internal geometry of the kagome lattice is determined based on the requirement of minimum actuation effort. Using non-dimensional parameters, the slenderness ratio of the kagome lattice can be defined as the quotient between the length of the hexagonal unit cell, shown in Fig. 5 and its height, i.e. $\lambda=\frac{\sqrt{3} d_{3}}{d_{1}}$. Also, the parameter $\beta=\frac{d_{1}}{d_{2}}$ is used for measuring the aspect ratio of an individual member. 
Figure 6 shows the variation of actuation force versus $\beta$ for different values of $\lambda$. It can be observed that for a kagome lattice with a defined slenderness ratio $\lambda$, the actuation effort decreases significantly with reducing member's thickness. On the other hand, increasing the member's length for a defined ratio of height to thickness reduces the actuation effort for in-plane bending deformation.

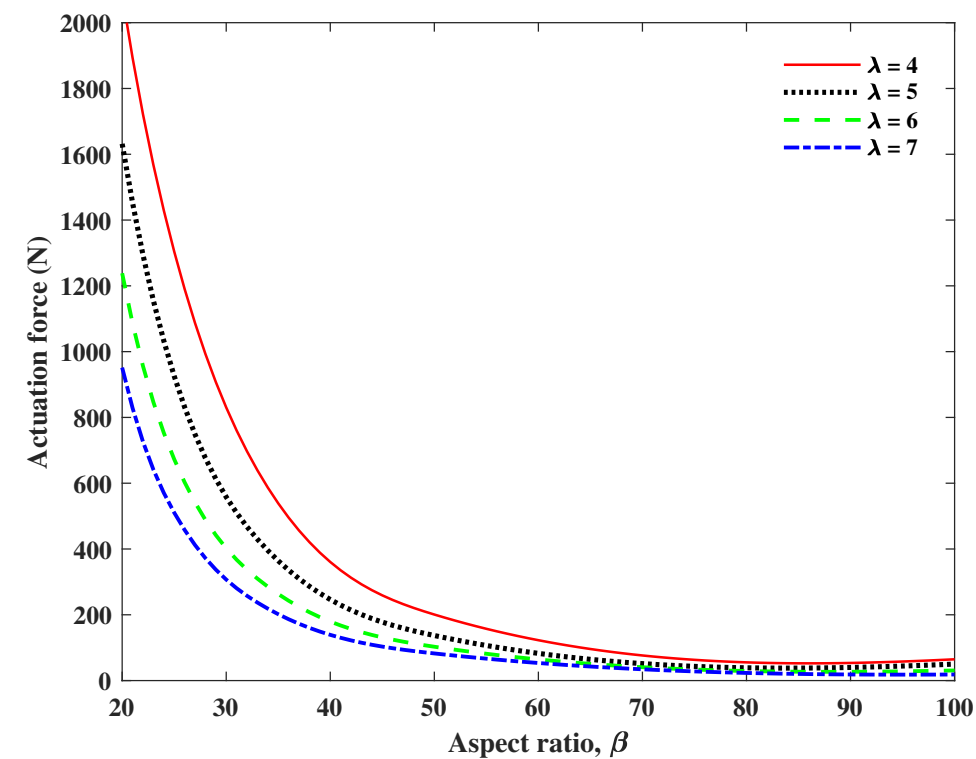

Figure 6: Variation of actuation force versus aspect ratio, $\beta=\frac{d_{1}}{d_{2}}$, for kagome lattices with different values of slenderness ratio, $\lambda=\frac{\sqrt{3} d_{3}}{d_{1}}$.

In many practical cases, the dimension $d_{3}$ is determined based on the design requirements for the distance between adjacent corridors, i.e., $\sqrt{3} d_{3}$. This work aims to make a lattice with a distance of $20 \mathrm{~mm}$ between corridors. From Fig. 6 it can be observed that for an aspect ratio greater than around 80, the asymptotic actuation force reaches its minimum value. On the other hand, from the previous work [8], the propensity to generate exclusively in-plane displacement modes can be ensured for a kagome lattice with an aspect ratio of $\approx 120$ and a slenderness ratio of $\approx 5.5$, in which out-of-plane compliant modes have higher levels of activation energy. This will be clarified further in Figs 10 and 11, where 
the confined corridor and alternating shapes are obtained by the summation of low-energy in-plane compliant modes. Ultimately, Table 1 shows the value of parameter $d_{3}$ and two other geometrical parameters, $d_{1-2}$ to ensure a lattice with the minimum required in-plane actuation force.

Table 1: Dimensions of lattice structure, where dimensions $d_{1-3}$ are in accordance with Fig.

\begin{tabular}{ccc}
\hline$d_{1}$ & $d_{2}$ & $d_{3}$ \\
\hline $3.62 \mathrm{~mm}$ & $30 \mu \mathrm{m}$ & $11.55 \mathrm{~mm}$ \\
\hline
\end{tabular}

\subsection{Compliant boundary condition}

The boundary conditions are designed to have minimum effect on the in-plane movement of the kagome lattice under large displacement values. This is accomplished by using leaf springs at boundary points as compliant boundary conditions, see Fig. 7. The leaf springs are designed from the same grade of steel as the structure.

Compliant boundary conditions are modelled as three longitudinal springs $K_{i}(i=1,2,3)$ and one torsional spring $K_{6}$. Denoting geometrical parameters of the leaf spring with $S_{1-3}$, as shown in Fig. 7, the equivalent stiffness in span $\left(K_{1}\right)$ and chord $\left(K_{2}\right)$ directions is calculated based on the deflection of a cantilever beam with zero slope at the tip point, where its movement is confined within the plan of the kagome lattice. The equivalent stiffness in normal direction $\left(K_{3}\right)$ is determined as the stiffness of bar element with the cross-sectional area of $S_{1} S_{2}$. The torsional stiffness $K_{6}$ is estimated based on the torsion constant $J$ given in Ref [18] for bars with rectangular cross section.

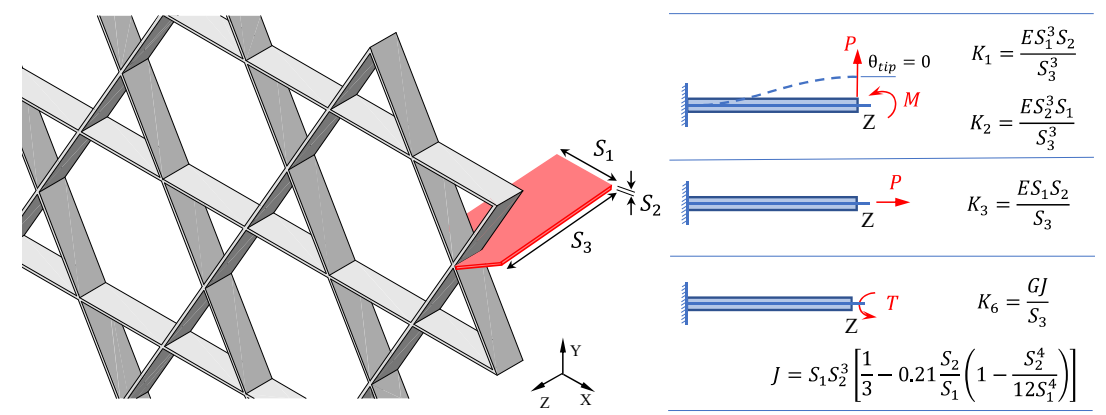


Figure 7: Modeling of compliant boundary condition with three longitudinal springs in three direction and one rotation spring normal to the plane.

From a practical point of view, the load is applied to the kagome lattice through some load leaf springs at the actuation points. More details on the

250 specific actuation mechanism can be found in Ref. [9]

Tables 2 and 3 give the dimension and equivalent stiffness values of standing and load leaf springs which are used in boundary conditions and load points, respectively. Standing leaf springs with a small thickness in the chord direction, $S_{2}$, provide a compliant boundary condition which allows the structure to move normal to the direction of actuation. Also, assigning a large value of $s_{1}$, and consequently a large stiffness $K_{1}$ in the span direction, results in a higher independence of actuating corridors. In addition, a high value of normal stiffness $K_{3}$ suppresses undesired out-of-plane deformation modes. It should be mentioned that the span-wise stiffness $K_{1}$ is neglected for load leaf springs which transfer span-wise movement to the lattice.

Table 2: Mechanical properties of leaf springs at boundary and load points, with notations presented in Fig. 7

\begin{tabular}{|c|ccc|}
\cline { 2 - 4 } \multicolumn{1}{c|}{} & $S_{1}$ & $S_{2}$ & $S_{3}$ \\
\hline Standing leaf spring & $6.35 \mathrm{~mm}$ & $50 \mu \mathrm{m}$ & $12 \mathrm{~mm}$ \\
\hline Load leaf spring & $6.35 \mathrm{~mm}$ & $50 \mu \mathrm{m}$ & $20 \mathrm{~mm}$ \\
\hline
\end{tabular}

Table 3: Equivalent stiffness of leaf springs at boundary and load points in FE model, calculated using the relations given in Fig. 7

\begin{tabular}{|c|cccc|}
\cline { 2 - 5 } \multicolumn{1}{c|}{} & $K_{1}$ & $K_{2}$ & $K_{3}$ & $K_{6}$ \\
\hline Standing leaf spring & $1.4 \times 10^{6} \mathrm{~N} . \mathrm{m}^{-1}$ & $85{\mathrm{~N} . \mathrm{m}^{-1}}^{-1}$ & $4.9 \times 10^{6}{\mathrm{~N} . \mathrm{m}^{-1}}^{-1}$ & $1.6 \mathrm{~N} . \mathrm{mm}$ \\
\hline Load leaf spring & 0 & $18{\mathrm{~N} . \mathrm{m}^{-1}}^{-1}$ & $2.9 \times 10^{6} \mathrm{~N}^{-m^{-1}}$ & $1 \mathrm{~N} . \mathrm{mm}$ \\
\hline
\end{tabular}

With the geometry and constraints given above, Fig. 8 illustrates the performance of the designed compliant boundary conditions in comparison with built-in boundary conditions in reducing the actuation forces under large deformation. This figure also compares the actuation forces obtained by the linear and geometrically nonlinear analyses. The significant difference between the two solutions, which is observed from the early stages of displacement, illustrates 
the necessity of employing the nonlinear formulation even for small-amplitude actuation.

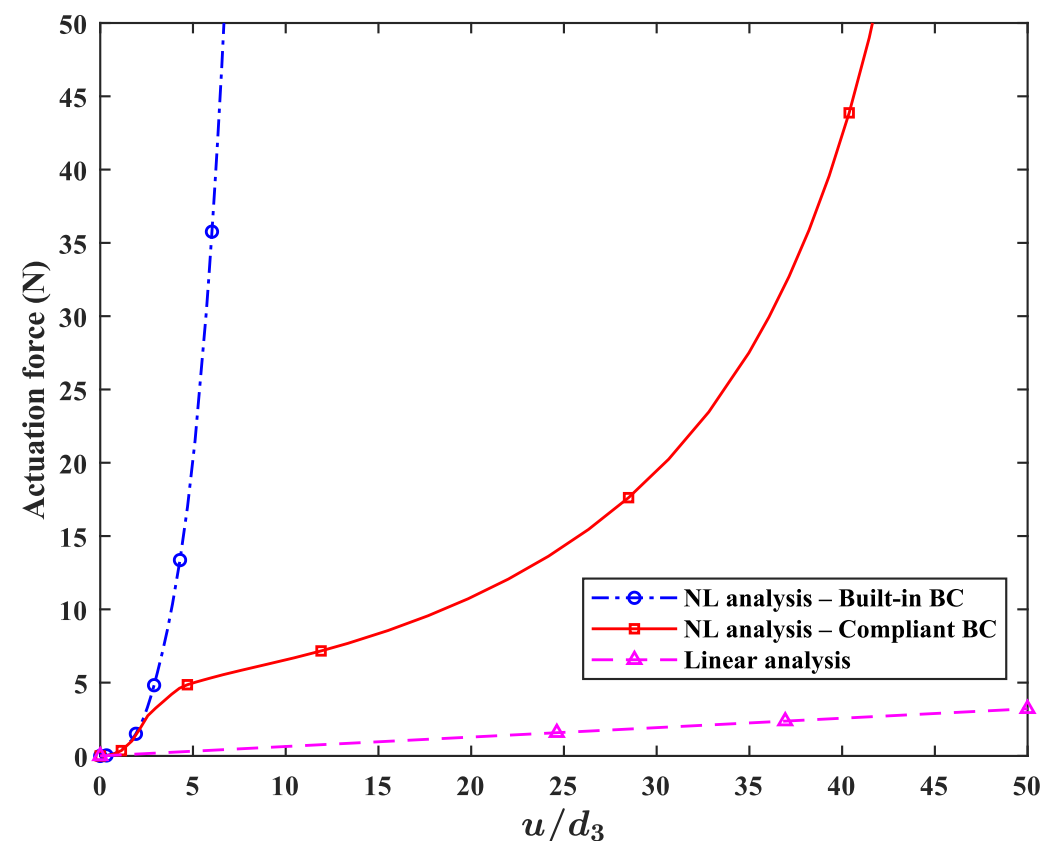

Figure 8: Comparison of force-displacement responses of kagome lattice under alternating load condition obtained via linear and geometrically nonlinear FE analysis with built in and compliant boundary conditions

\subsection{Modal optimization}

In the case of the kagome lattice structure, we aim to produce a continuous structure capable of producing in-plane confined corridor modes. As per the model reduction technique, the desired deformation is invoked by summation of the compliance modes with low energy. Based on the initial stiffness matrix for the undeformed configuration, the compliance modes of the finite kagome lattice are obtained from Eq 22 and the six lowest energy modes are shown in Fig. 9. It should be mentioned that the low energy out-of-plane modes are avoided by applying the boundary conditions presented in Section 4.2. 


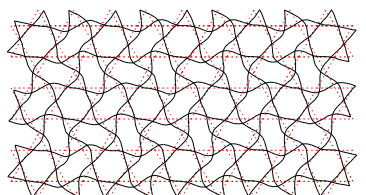

(a) Mode 1

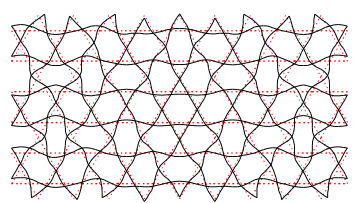

(d) Mode 4

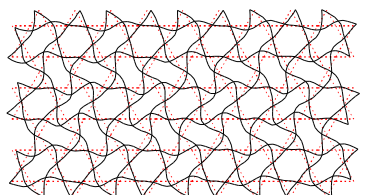

(b) Mode 2

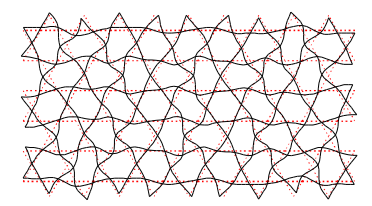

(e) Mode 5

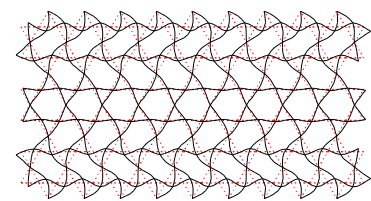

(c) Mode 3

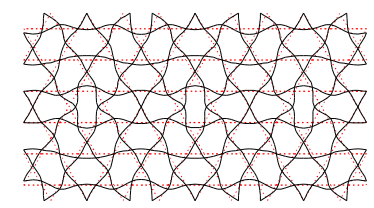

(f) Mode 6

Figure 9: First mode shapes of kagome lattice calculated based on the initial configuration that a desired displacement $q$ can be delivered, can be found as follows

$$
\overbrace{\left.\left[\boldsymbol{\Phi}_{r}\right]\right\}\{\boldsymbol{r}\}}^{(n \times c)}=\overbrace{[\boldsymbol{q}]}^{(c \times 1)}
$$

In this equation, $\boldsymbol{\Phi}_{r}$ is a subset of the compliance modal matrix in Eq 22 that contains the $c$ lowest required energy modes and $r$ shows their corresponding weighting coefficients.

The required compliance mode shapes and the corresponding weights for producing confined corridor mode shapes are given in Table 4. Figure 10 shows the resultant summation of the weighted compliance modes. As can be expected, the symmetry in the geometry of the lattice is reflected in the repeated modes and weight coefficients in Table 4 .

Also, the alternating deformed shape, according what is desired in Fig. 5, would be obtained by summation of mode shape 5 with weight 0.824 and mode shape 10 with weight 0.182, shown in Fig. 11.

At this point, it can be observed that the presented kagome lattice with aspect ratio of $\beta \approx 120$ and slenderness ratio of $\lambda \approx 5.5$ under the defined 
Table 4: Weighting coefficients to produce the corridor modes

\begin{tabular}{|l|c|c|c|c|c|c|}
\cline { 2 - 7 } \multicolumn{1}{c|}{} & Mode 5 & Mode 6 & Mode 10 & Mode 12 & Mode 17 & Mode 18 \\
\hline Corridor mode 1 & 0.611 & -0.721 & 0.788 & 0.513 & -0.558 & 0.000 \\
\hline Corridor mode 2 & -0.736 & 0.521 & 0.000 & 0.514 & -0.948 & 0.682 \\
\hline Corridor mode 3 & 0.714 & 0.000 & -0.591 & -0.516 & 0.000 & 0.913 \\
\hline Corridor mode 4 & -0.714 & 0.000 & 0.591 & -0.516 & 0.000 & 0.913 \\
\hline Corridor mode 5 & 0.736 & 0.521 & 0.000 & 0.514 & 0.948 & 0.682 \\
\hline Corridor mode 6 & -0.611 & -0.721 & -0.788 & 0.513 & 0.558 & 0.000 \\
\hline
\end{tabular}

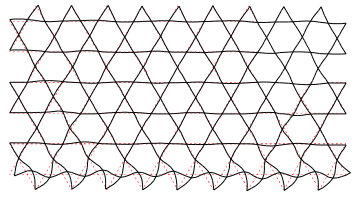

(a) Corridor mode 1

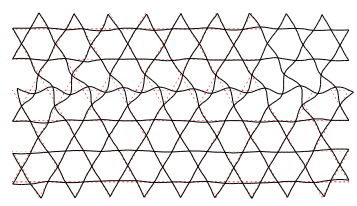

(a) Corridor mode 4

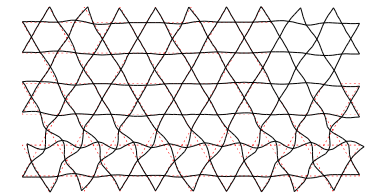

(a) Corridor mode 2

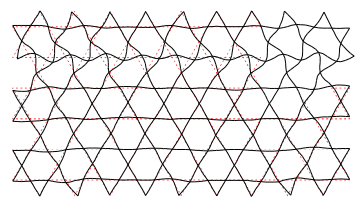

(a) Corridor mode 5

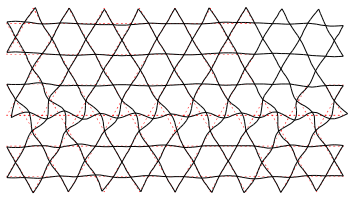

(a) Corridor mode 3

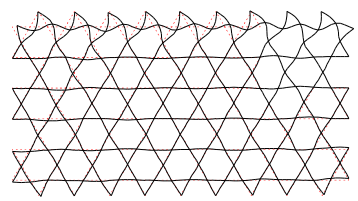

(a) Corridor mode 6

Figure 10: Confined corridor shapes obtained by summation of compliant mode shapes weighted according Tables 4

actuation corridor as well as alternating mode shapes with summation of compliance modes with low energy. However, under large deformation the stiffness matrix and consequently the compliance modes change. The change of compliant modes during deformation can be quantified using the Modal Assurance Criterion (MAC) [19] defined as

$$
\operatorname{MAC}\left({ }^{0} \boldsymbol{\psi}^{i},{ }^{t} \boldsymbol{\psi}^{i}\right)=\frac{\left({ }^{0} \boldsymbol{\psi}^{i^{T} t} \boldsymbol{\psi}^{i}\right)^{2}}{\left({ }^{0} \boldsymbol{\psi}^{i^{T} 0} \boldsymbol{\psi}^{i}\right)\left({ }^{t} \boldsymbol{\psi}^{i^{T} t} \boldsymbol{\psi}^{i}\right)}
$$

where ${ }^{0} \boldsymbol{\psi}^{i}$ and ${ }^{t} \boldsymbol{\psi}^{i}$ are the $i$-th compliant mode of the compliant structure at the initial configuration and time $t$, respectively. The parameter is calculated for the first six mode shapes under the applied displacement $u / d 3=10 \%$. The compliance modes are illustrated in Fig. 12, and the corresponding MAC values 


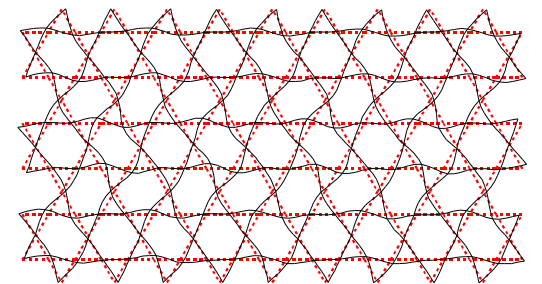

(a) Mode 5

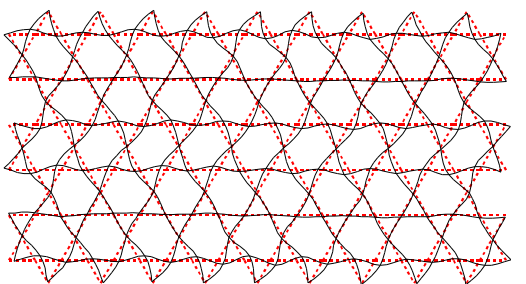

(b) Mode 10

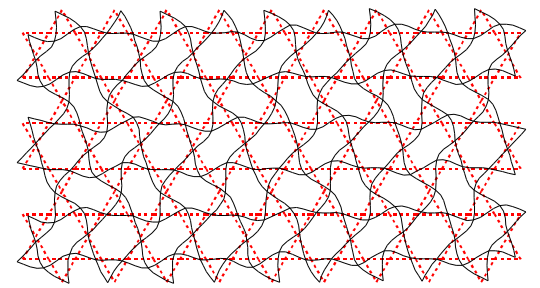

(c) Resultant alternative mode shape

Figure 11: Two compliant mode shapes 5 and 10 and the resultant alternating mode shape, obtained by component weights 0.824 and and weight 0.182 , respectively.

are given in Table 5 .

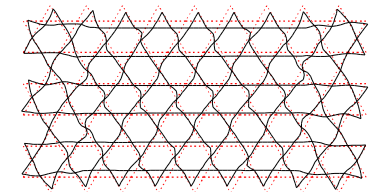

(a) Mode 1

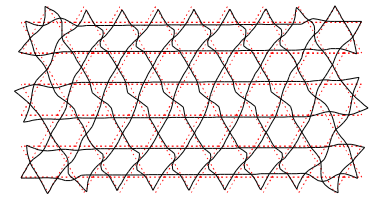

(d) Mode 4

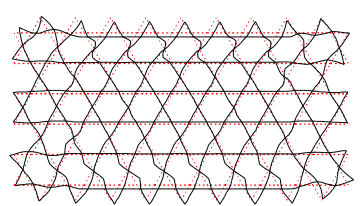

(b) Mode 2

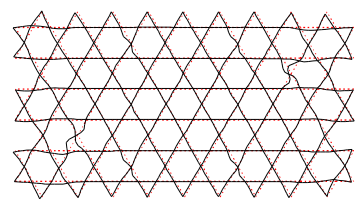

(e) Mode 5

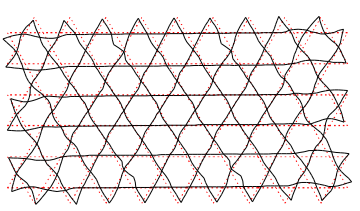

(c) Mode 3

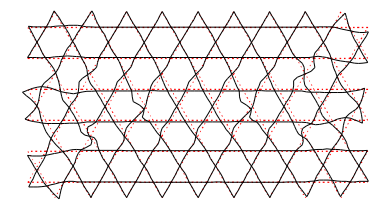

(f) Mode 6

Figure 12: First mode shapes of kagome lattice under applied displacement $u / d_{3}=10 \%$

Table 5: Modal Assurance Criterion for six first mode shapes under applied displacement $u / d_{3}=10 \%$

\begin{tabular}{|c|c|c|c|c|c|c|}
\cline { 2 - 7 } \multicolumn{1}{c|}{} & Mode 1 & Mode 2 & Mode 3 & Mode 4 & Mode 5 & Mode 6 \\
\hline MAC\% & 7.8 & 2.2 & 1.8 & 5.4 & 0.3 & 0.3 \\
\hline
\end{tabular}

The significant change in compliant mode shapes is due to tightening of internal members under large deformation. Figure 13 compares the deformed shapes 
of the kagome lattice under displacement value of $u / d_{3}=10 \%$ obtained from nonlinear and linear analyses. Importantly, the tightening of internal members causes a significantly large increase in actuation force, as shown in Fig. 8, in comparison with the linear solution that ignores the additional stiffness induced by the stress state. The tightening of internal members also causes a lateral shrinkage deformation toward the center of the kagome lattice structure in Fig. 13(b). To mitigate the tightening problem, some internal members can be removed. This implies the necessity of optimizing the kagome lattice design to find an optimum continuous lattice with the aim of reducing actuation force under large deformation.

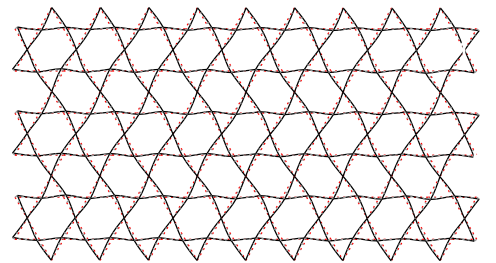

Figure 13: Kagome lattice under applied displacement $u / d_{3}=10 \%$ obtained via linear and

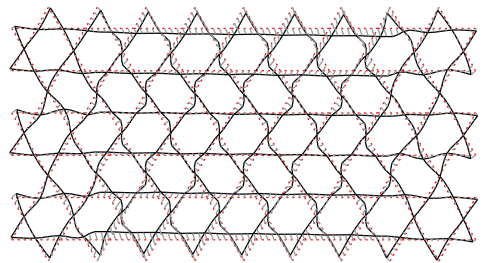

Figure 13: Kagome lattice under applied displacement $u / d_{3}=10 \%$ obtained via linear and geometrically nonlinear analysis

As can be observed in Eqs (15b) and (15c), the nonlinear stiffness matrix and the internal force vector are determined based on the instantaneous stress field in each increment. By removing each member of the lattice, the stress distribution changes in the new structure which in turn will change the incremental deformation path of the lattice under large amplitude actuation. As per the large numbers of configurations that can be obtained by removing different members of the lattice as well as the nonlinear deformation path which each candidate configuration will undergo, the optimum design is obtained using Bayesian optimization [20], which is a class of machine-learning-based optimization methods. Bayesian optimization, as a black-box derivative-free global optimization, provides a powerful tool in the development of structurally efficient lattice design under large deformation. 

equal to the total number of lattice members, $m$, and the existence and absence of each member is represented by 0 or 1 as 


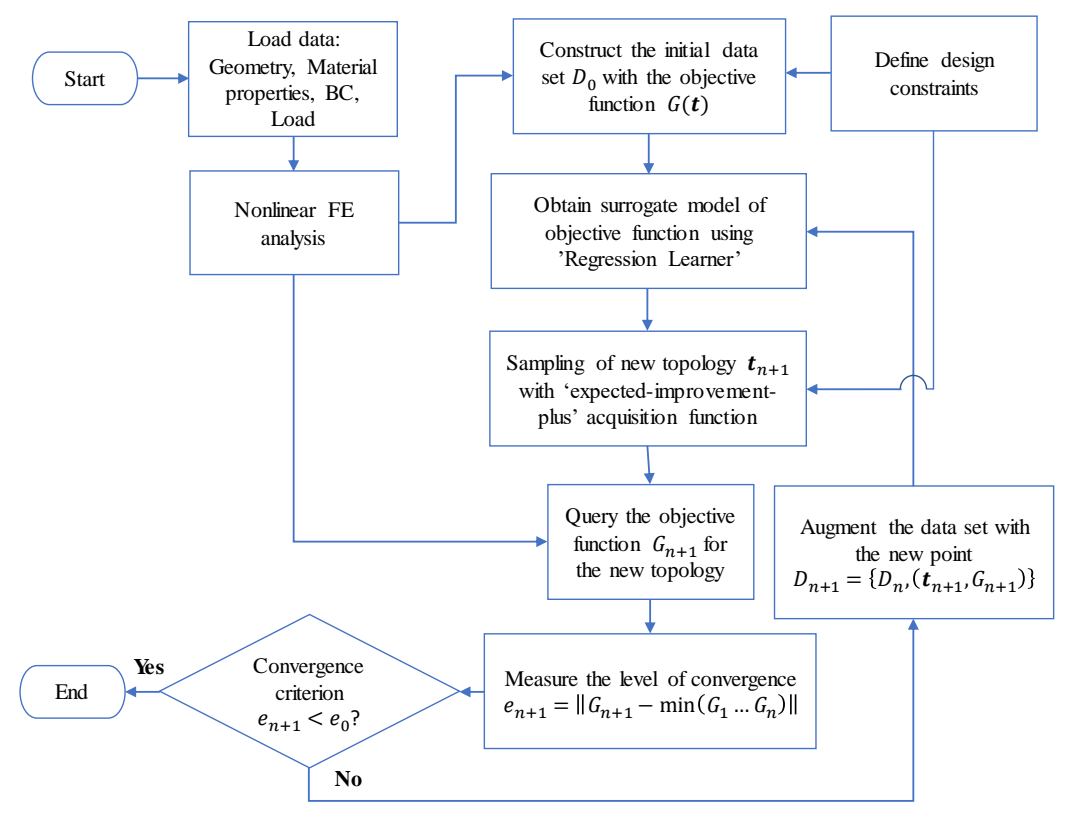

Figure 14: Flowchart of the proposed optimization algorithm

$$
t_{i}= \begin{cases}1 & \text { if member } i \text { exists in the lattice } \\ 0 & \text { otherwise }\end{cases}
$$

Note that the topology vector defines only the existence of members in the lattice. The size of members in the topology vector are the same and are defined based on the design criteria as discussed in Section 4.1.

\subsection{Definition of constraints}

In order to guarantee feasibility of the optimal lattice as an integrated actuating structure, some constraints are defined on the topology of lattice within the design domain.

First, a candidate topology should be symmetric with respect to the middle column of the lattice. i.e. column $c_{10}$ in Fig. 5. Indeed, we are interested to find the optimal design under the alternating load condition, in which load direction changes from right to left (or from left to right) between two neighbouring corridors. In other words, the obtained solution should be the optimum design 
under the applied load condition with left direction on the rows $r_{1,3,5}$ and right direction on rows $r_{2,4,6}$, as shown in Fig. 5, as well as the opposite applied load with left direction on the rows $r_{2,4,6}$ and right direction on rows $r_{1,3,5}$. With regard to the symmetric location of boundary and load points, a symmetric geometry ensures that the solution will remain optimum with reversing the load direction at neighbouring corridors.

The remaining constraints are categorized in two classes - the constraints (1) along the corridors and (2) at joints between corridors defined as follows:

1. Constraints along each corridor: These constraints are applied to transfer loads along each corridor as active members.

1.1. A member placed at a load point can not be removed (i.e., members on columns $c_{4}, c_{10}$ and $c_{16}$ along each corridor at Fig. 5).

1.2. Between two line loads only one member can be removed.

2. Constraints at joints: This constraint aims to keep the structural integrity of the lattice at joints between two neighbouring corridors. If four crossing members at a typical joint are denoted by $t_{a}, t_{b}, t_{c}$ and $t_{d}$ as shown in Fig. $15(\mathrm{a})$, then the joint constraint is defined as

$$
\left(t_{a}+t_{b}\right) \cdot\left(t_{c}+t_{d}\right) \neq 0
$$

Three typical feasible joint topologies are illustrated in Fig. 15(b)-(d). Note that the grey members indicate removed members with $t_{i}=0$ in the topology vector.

As an illustration, Fig. 16 presents constraints 1 and 2 for feasible topologies on a small part of the studied kagome lattice in Fig. 5.

\subsection{Optimum kagome lattice for producing large amplitude in-plane deforma-} tion

Figure 17 shows the obtained optimum design for the finite lattice and the total actuation force versus displacement for the optimum lattice obtained via geo- 


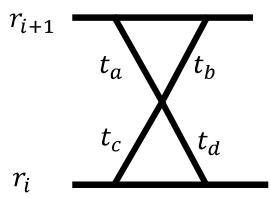

(a)

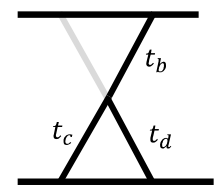

(b)

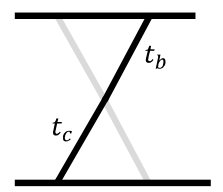

(c)

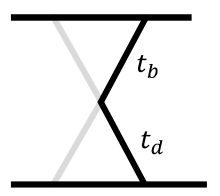

(d)

Figure 15: (a) A sample joint with four members between two corridors, and three typical feasible joint topologies related to (b): $t_{a}=0$, (b): $t_{a}=t_{d}=0$ and (b): $t_{a}=t_{c}=0$ in Eq (26)

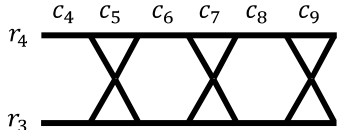

(a)

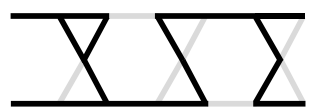

(b)

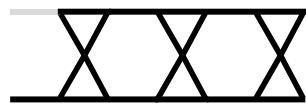

(c)

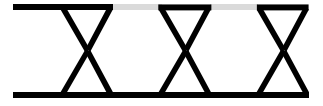

(d)

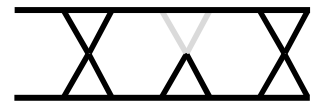

(e)

Figure 16: Constraints for feasible topologies: (a) a sample part of studied kagome lattice in Fig. 5 containing columns $c_{4}-c_{9}$ of corridors $r_{3}-r_{4}$ and, (b) a typical feasible topology, infeasible topologies: (c) violating constraint 1.1 (removing member under load) (d) violating constraint 1.2 (removing two members between two line loads, which makes the member on row $r_{4}$ and column $c_{7}$ ineffective) and (d) violating constraint 2 (loosing the connection between two corridors)

metrically nonlinear analysis. It can be observed that by the effective release of the internal stress field in the structure, the optimum lattice gives the significant reduction of two orders of magnitude in the actuation force from $1240 \mathrm{~N}$ to 80 $\mathrm{N}$ at the displacement of $u / d_{3}=50 \%$.

Figure 18 shows the deformed shapes of the optimum kagome lattice obtained by nonlinear FE analysis under alternating applied displacements at four different values, with equal intervals of $u / d_{3}=10 \%$. In comparison with Fig 13(b) at $u / d_{3}=10 \%$, the vertical shrinkage has been eliminated in the deformed shape of optimum kagome lattice at Fig 18(a). Due to the boundary condition discontinuities at the top and bottom of the kagome lattice, the boundary areas shrink in the vertical direction with increasing actuation amplitude. However, the inner corridors are still capable of generating the large amplitude alternating deformed shape, as shown in Fig 18(b)-(d). 


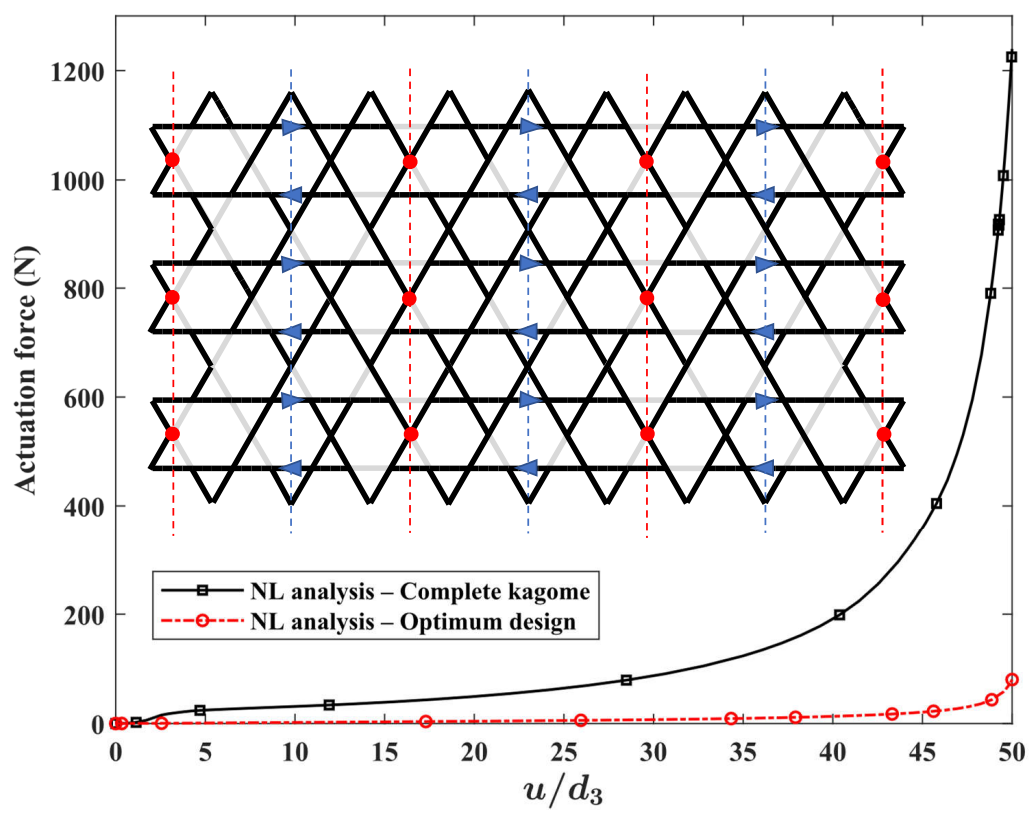

Figure 17: Nonlinear force-displacement diagrams under alternating applied load for the optimum lattice in comparison with the complete kagome lattice. The grey members in the optimal design of kagome lattice indicate the removed members.

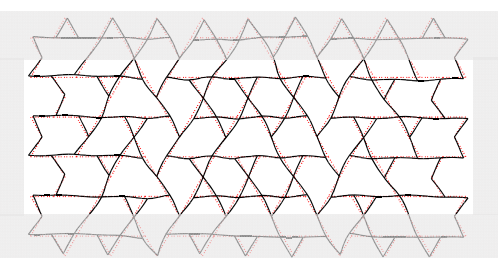

(a) $u / d_{3}=10 \%$

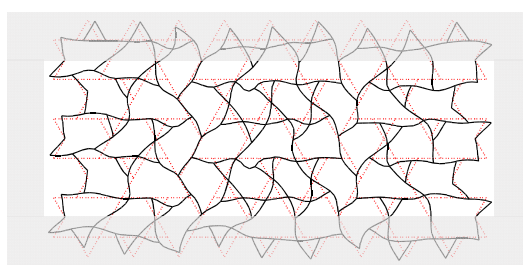

(c) $u / d_{3}=30 \%$

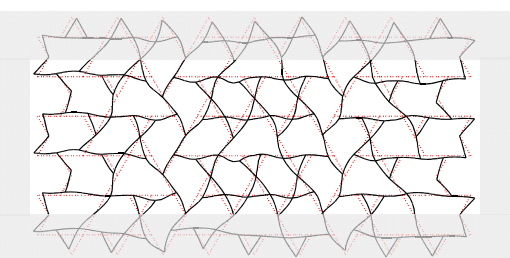

(b) $u / d_{3}=20 \%$

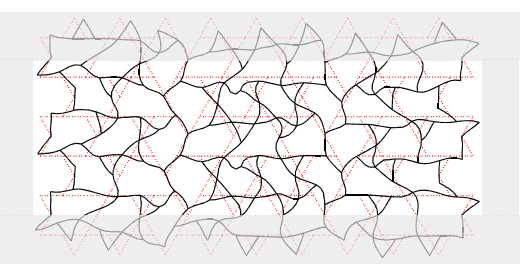

(d) $u / d_{3}=40 \%$

Figure 18: Deformed shape of the inner part of the optimum kagome lattice under large amplitude alternating actuation. With increasing the actuation amplitude, the top and bottom boundary areas shrink in vertical direction due to boundary condition discontinuities.

\section{Conclusions}

This paper has demonstrated the determination and enhancement of compliant modes for adaptive beam assemblies - with a particular focus on the kagome 
lattice geometry. As a continuous system with many degrees of freedom, determination of compliance modes are carried out based on finite element discretization method and then singular value decomposition of stiffness matrix. Finite element analysis is performed using an in-house FE code developed based on the elaborated second-order nonlinear beam finite element formulation presented for beam assemblies under large rotations. Adaptivity of the structure under large deformation has been also improved by introducing compliant boundary conditions. Using a reduced modal model as the basis for the design of adaptive structure, the desired actuation mode is constructed by weighted summation of small number of key modes with low energy. Conversely, the locations of boundary points are specified to suppress undesired out-of-plane compliant mode shapes.

${ }_{425}$ With increasing deformation, the induced internal stress state causes change of the compliant mode shapes which are substantial and reduce the efficiency of designed structure for delivering the high amplitude actuation. This implies the necessity of re-optimization of the designed adaptive structure for large actuating values. This work employs the Bayesian optimization algorithm, as a black-box derivative-free global optimization tool for the design of effective lattice. The optimization problem is defined with the complete kagome lattice as the largest design space and assumes only the removal of members. Also, some constraints are defined on removed members to guarantee feasibility of the optimal lattice as an integrated actuating structure. The process detailed yields an optimum design, in which the internal stress is released effectively, with the significant reduction of two orders of magnitude in actuation force.

In continuing work a full-scale actuated module is being fabricated and will be used to validate the nonlinear dynamic response of the optimized lattice. This adaptive surface will form the basis of a large scale flow control experiment in 440 Imperial College's $10 \times 5$ wind tunnel. 


\section{Acknowledgments}

The authors would like to show their gratitude to the Engineering and Physical Sciences Research Council which provided financial support, under agreement EP/R032467/1.

\section{Appendix}

The FE formulation of the nonlinear beam element is implemented using the linear interpolations $\left(h_{1}\right.$ and $h_{2}$ in Eqs $\left.(27)\right)$ to describe axial displacement and torsional rotation, and Hermitian functions $\left(h_{i} ; i=3 . .6\right.$ in $\left.\operatorname{Eqs}(27)\right)$ to interpolate the transverse bending displacements and rotations.

$$
\begin{aligned}
& h_{1}=1-\frac{x_{3}}{L} \\
& h_{2}=\frac{x_{3}}{L} \\
& h_{3}=1-3 \frac{x_{3}^{2}}{L^{2}}+2 \frac{x_{3}^{3}}{l^{3}} \\
& h_{4}=x_{3}\left(1-2 \frac{x_{3}}{L}+\frac{x_{3}^{2}}{l^{2}}\right) \\
& h_{5}=\frac{x_{3}^{2}}{L^{2}}\left(3-2 \frac{x_{3}}{l}\right) \\
& h_{6}=\frac{x_{3}^{2}}{L}\left(-1+\frac{x_{3}}{l}\right)
\end{aligned}
$$

\section{References}

[1] S. Pellegrino, C. R. Calladine, Matrix analysis of statically and kinematically indeterminate frameworks, International Journal of Solids and Structures 22 (4) (1986) 409-428.

[2] D. B. McCallen, K. Romstad, A continuum model for the nonlinear analysis of beam-like lattice structures, Computers \& structures 29 (2) (1988) 177197. 
[3] S. Cao, M. Huo, N. Qi, C. Zhao, D. Zhu, L. Sun, Extended continuum model for dynamic analysis of beam-like truss structures with geometrical nonlinearity, Aerospace Science and Technology (2020) 105927.

[4] L. Chen, L. A. Beex, P. Z. Berke, T. J. Massart, S. P. Bordas, Generalized quasicontinuum modeling of metallic lattices with geometrical and material nonlinearity and variability, Computer Methods in Applied Mechanics and Engineering 366 (2020) 112878.

[5] M. Jamshidian, N. Boddeti, D. Rosen, O. Weeger, Multiscale modelling of soft lattice metamaterials: micromechanical nonlinear buckling analysis, experimental verification, and macroscale constitutive behaviour, International Journal of Mechanical Sciences (2020) 105956.

[6] A.-J. Wang, D. McDowell, In-plane stiffness and yield strength of periodic metal honeycombs, Journal of engineering materials and technology 126 (2) (2004) 137-156.

[7] N. Wicks, S. Guest, Single member actuation in large repetitive truss structures, International Journal of Solids and Structures 41 (3-4) (2004) 965978.

[8] J. Bird, M. Santer, J. Morrison, The determination and enhancement of compliant modes for actuation in structural assemblies, International Journal of Solids and Structures 106 (2017) 264-273.

[9] J. Bird, M. Santer, J. Morrison, Compliant kagome lattice structures for generating in-plane waveforms, International Journal of Solids and Structures 141 (2018) 86-101.

[10] J. Bird, M. Santer, J. Morrison, Experimental control of turbulent boundary layers with in-plane travelling waves, Flow Turbulence and Combustion 100 (4) (2018) 1015-1035.

[11] M. Epsteini, D. W. Murray, Large deformation in-plane analysis of elastic beams, Computers and Structures 6 (1) (1976) 1-9. 
[21] Matlab machine learning and deep learning toolbox, The MathWorks Inc. (R2019a).

[12] A. J., An excursion into large rotations, Computer methods in applied mechanics and engineering 32 (1-3) (1982) 85-115.

[13] S. Kordkheili, Z. Soltani, A layerwise finite element for geometrically nonlinear analysis of composite shells, Composite Structures 186 (2018) 355364.

[14] K. G. Soltani Z, Kordkheili SH, Experimental and numerical study of geometrically nonlinear behavior of corrugated laminated composite shells using a nonlinear layer-wise shell fe formulation, Engineering Structures 184 (2019) 61-73.

[15] K. Bathe, S. Bolourchi, Large displacement analysis of three-dimensional beam structures, International journal for numerical methods in engineering, vol. 14, (1979) 14 (1979) 961-986.

[16] T. J. R. Hughes, The finite element method: linear static and dynamic finite element analysis, Dover, New York City, 1987.

[17] K. J. Bathe, Finite element procedures, Prentice-Hall, Upper Saddle River, New Jersy, 1996.

[18] W. C. Young, R. G. Budynas, Roark's Stress \& Strain Formulas, 7thedition mac-graw hill Publication, 2002.

[19] D. Ewins, Modal testing: theory, practice and application, 2000, Research Studies Press LTD., Baldock, Hertfordshire, England, 2000.

[20] P. I. Frazier, A tutorial on bayesian optimization, arXiv preprint arXiv:1807.02811 (2018). 